\title{
Transcranial Alternating Current Stimulation Attenuates Neuronal Adaptation
}

\author{
Kohitij Kar, Jacob Duijnhouwer, and ๑Bart Krekelberg \\ Center for Molecular and Behavioral Neuroscience, Rutgers University, Newark, New Jersey 07102
}

We previously showed that brief application of $2 \mathrm{~mA}$ (peak-to-peak) transcranial currents alternating at $10 \mathrm{~Hz}$ significantly reduces motion adaptation in humans. This is but one of many behavioral studies showing that weak currents applied to the scalp modulate neural processing. Transcranial stimulation has been shown to improve perception, learning, and a range of clinical symptoms. Few studies, however, have measured the neural consequences of transcranial current stimulation. We capitalized on the strong link between motion perception and neural activity in the middle temporal (MT) area of the macaque monkey to study the neural mechanisms that underlie the behavioral consequences of transcranial alternating current stimulation. First, we observed that $2 \mathrm{~mA}$ currents generated substantial intracranial fields, which were much stronger in the stimulated hemisphere $(0.12 \mathrm{~V} / \mathrm{m})$ than on the opposite side of the brain $(0.03 \mathrm{~V} / \mathrm{m})$. Second, we found that brief application of transcranial alternating current stimulation at $10 \mathrm{~Hz}$ reduced spike-frequency adaptation of MT neurons and led to a broadband increase in the power spectrum of local field potentials. Together, these findings provide a direct demonstration that weak electric fields applied to the scalp significantly affect neural processing in the primate brain and that this includes a hitherto unknown mechanism that attenuates sensory adaptation.

Key words: entrainment; local field potential; motion adaptation; motion after effect; neural mechanisms; transcranial alternating current stimulation

\section{Significance Statement}

Transcranial stimulation has been claimed to improve perception, learning, and a range of clinical symptoms. Little is known, however, how transcranial current stimulation generates such effects, and the search for better stimulation protocols proceeds largely by trial and error. We investigated, for the first time, the neural consequences of stimulation in the monkey brain. We found that even brief application of alternating current stimulation reduced the effects of adaptation on single-neuron firing rates and local field potentials; this mechanistic insight explains previous behavioral findings and suggests a novel way to modulate neural information processing using transcranial currents. In addition, by developing an animal model to help understand transcranial stimulation, this study will aid the rational design of stimulation protocols for the treatment of mental illnesses, and the improvement of perception and learning.

\section{Introduction}

Transcranial alternating current stimulation (tACS) produces acute perceptual (Strüber et al., 2014), motor (Joundi et al., 2012), and cognitive (Sela et al., 2012) changes in humans. There is, however, a paucity of studies probing the neural underpin-

\footnotetext{
Received July 10, 2016; revised Nov. 12, 2016; accepted Dec. 17, 2016.

Author contributions: K.K. and B.K. designed research; K.K. and J.D. performed research; K.K. and B.K. analyzed data; K.K., J.D., and B.K. wrote the paper.

This work was supported by Army Research Office W911NF-14-1-0408, National Institute of Mental Health, National Institute of Neurological Disorders and Stroke R01MH111766, Eye Institute of the National Institutes of Health R01EY017605, and the Charles and Johanna Busch Memorial Fund at Rutgers, State University of New Jersey. We thank Denis Paré for helpful discussions; and Anne McCormick and Jasmine Siegel for excellent technical support. The authors declare no competing financial interests.

Correspondence should be addressed to Dr. Kohitij Kar, Center for Molecular and Behavioral Neuroscience, Rutgers University, 197 University Avenue, Newark, NJ 07102. E: kohitij@vision.rutgers.edu.

DOI:10.1523/JNEUROSCI.2266-16.2016

Copyright $\odot 2017$ the authors $\quad 0270-6474 / 17 / 372325-11 \$ 15.00 / 0$
}

nings of these effects in the intact brain (Herrmann et al., 2013; Reato et al., 2013), and the influence of tACS on neural activity remains poorly understood. As a consequence, development of this promising technique for noninvasive neuromodulation proceeds mainly by trial and error.

To start addressing this issue, we developed a nonhuman primate model to directly measure neural consequences of tACS. Finite element current flow models suggest that the strength and orientation of intracranial fields depend both on the gross anatomical structure as well as the convolutions of the brain (Wagner et al., 2007; Datta et al., 2009; Dmochowski et al., 2011). Based on the structural similarities of the macaque and human brain, we therefore expect that a macaque model results in a better approximation of current flows in humans than a lissencephalic rodent brain. Moreover, the functional similarities between the human and nonhuman primate, especially in their highly devel- 
oped visual systems (Orban et al., 2003), maximizes the translational validity of this animal model.

We have recently shown that tACS at $10 \mathrm{~Hz}$ counteracts the behavioral effects of motion adaptation in humans (Kar and Krekelberg, 2014). This phenomenon is important for several reasons. First, unlike the typical long periods (tens of minutes) used in transcranial direct current stimulation, the attenuation of adaptation occurs even for brief periods of stimulation (seconds). This has practical benefits and attests to the potency of this method of noninvasive neuromodulation. Second, because the phenomenon is difficult to explain using only the known mechanisms of transcranial current stimulation (e.g., increased/decreased excitability, or the entrainment of neural oscillations), we hypothesize that it could rely on a different, hitherto unknown neural consequence of tACS.

To understand the neural basis of the attenuation of motion adaptation by tACS, we focused on the middle temporal area (MT) of the macaque monkey. Neurons in area MT are exquisitely sensitive to visual motion (Born and Bradley, 2005), and prolonged exposure to a moving stimulus (i.e., sensory adaptation to motion) significantly changes their response properties (Priebe et al., 2002; Kohn and Movshon, 2004; Krekelberg et al., 2006; Schlack et al., 2007; Kar and Krekelberg, 2016). Given the close link between neural activity in area MT and the percept of motion (Parker and Newsome, 1998), understanding neural changes induced by tACS in area MT will help to understand how tACS affects behavior.

We recorded extracellular potentials in area MT of awake macaques while applying tACS using scalp electrodes. We first confirmed that weak currents applied to the scalp induced significant intracranial electric fields. Then, we investigated changes in neural activity reflected in individual neurons' firing rates and the synchronous population activity reflected in the local field potentials (LFPs). In both measures of neural activity, we found evidence that the effect of adaptation was partially undone by tACS. This provides direct support for our hypothesis of a novel mechanism by which tACS affects behavior: the attenuation of neural adaptation.

\section{Materials and Methods}

The experiments involved 2 adult, male rhesus macaques (Macaca mulatta). Experimental and surgical protocols were approved by the Rutgers University Animal Care and Use Committee and complied with guidelines for the humane care and use of laboratory animals of the National Institutes of Health.

\section{Surgical procedures and recording electrode location}

All surgical procedures were conducted under sterile conditions using isoflurane anesthesia. Titanium head posts (Gray Matter Research) were attached to the skull using titanium bone screws. Custom-made highdensity polyethylene recording chambers were implanted normally to the skull, and dorsal to the expected location of MT. We confirmed recording locations in area MT on the basis of structural magnetic resonance images obtained after implantation, as well as on the basis of physiological criteria such as the high prevalence of direction selective responses, and the relatively small receptive fields (compared with the neighboring medial superior temporal dorsal area).

\section{Recording}

Visual stimulus generation, the triggering of tACS, and data acquisition were under the control of in-house software for visual experimentation (Neurostim, http://neurostim.sourceforge.net). Stimuli were presented on a CRT monitor (Sony GDM-520) spanning $30^{\circ} \times 40^{\circ}$ at a resolution of $1024 \times 768$ pixels and a refresh rate of $150 \mathrm{~Hz}$. At the beginning of each recording session, we punctured the dura with a sharp, metal guide tube to allow access to the cortex. The guide tube or one of the head screws served as the ground for the electrode signal. We used a micro-positioner (NAN Instruments) to lower parylene-coated tungsten electrodes (standard profile; $200 \mu \mathrm{m}$ shank diameter; $1.5 \mathrm{M} \Omega$; FHC) into area MT through the guide tube.

We manually isolated single cells by listening to their visually driven response, which was made audible on a speaker while the monkey observed moving stimuli (see Experimental procedures). The raw signal was sampled at $25 \mathrm{kHz}$ using Alpha Lab (Alpha-Omega Engineering). To extract spikes, we first bandpass filtered the raw signal between $300 \mathrm{~Hz}$ and $6 \mathrm{kHz}$, and then applied a threshold equal to 4 SDs of the filtered signal. We used KlustaKwik (Harris et al., 2000; Kadir et al., 2014) to cluster these waveforms into separate units (up to 3, significantly direction tuned units per recording depth). LFPs were extracted from the raw signal by bandpass filtering between 1 and $120 \mathrm{~Hz}$ and then resampling at $781.25 \mathrm{~Hz}$. Eye movements were recorded using an infrared video eye tracker (Eyelink 1000; SR Research). Trials in which eye position deviated from the fixation point by $>1^{\circ}$ were not used in the analysis.

\section{Transcranial stimulation}

Matching the procedures we previously used in humans (Kar and Krekelberg, 2012, 2014; Wright and Krekelberg, 2014), we delivered tACS with an STG4002 stimulus generator (Multi Channel Systems) through $3.2 \mathrm{~cm} \times$ $3.2 \mathrm{~cm}$ reusable surface electrodes (BML Basic Physician's Supply).

We performed extensive tests on the stimulator to ensure its reliable operation. In the software controlled off-state, the current (through a $10 \mathrm{k} \Omega$ resistor; the maximum resistance of the stimulation circuit in the experiments) was measured to be 0 . Stimulation onset and offset time were confirmed to be within $20 \mu$ s of delivering a trigger pulse by our software. The trigger pulse was generated within one monitor frame (6.7 $\mathrm{ms}$ ) from the relevant visual stimulus onset (see below); and because a predefined current duration ( $3 \mathrm{~s}$ ) was used, tACS offset was also reliably within one monitor frame from the relevant visual stimulus offset. The applied current was always sinusoidal with a $2.0 \mathrm{~mA}$ peak-to-peak amplitude and a $10 \mathrm{~Hz}$ frequency. Because of the 14 bit digitization of the stimulator, the sinusoidal current waveform did not result in measurable higher harmonics. The voltage of the stimulator was monitored throughout the experiment. It was always $<20 \mathrm{~V}$ and never near the $120 \mathrm{~V}$ compliance voltage (i.e., the resistance of the entire stimulation circuit, including the head, was always $<10 \mathrm{k} \Omega$ ).

One tACS electrode was always placed $4 \mathrm{~cm}$ anterior to the vertex. In all experiments, a second electrode was placed between the ear and the recording chamber, adjacent to area MT (in the left hemisphere for Monkey $\mathrm{N}$ and in the right hemisphere for Monkey M). Stimulation through this electrode, ipsilateral to the recorded neurons, and the vertex resulted in the "local" conditions. Our intracranial field recordings (see Results) confirmed that this montage led to substantial electric fields in the area from which we recorded the neurons. In a subset of experiments, an additional electrode was placed in the mirrored position on the opposite hemisphere. Stimulation through this contralateral electrode and the vertex resulted in the "remote" conditions. In this montage, most current presumably flowed through the hemisphere from which we did not record neurons. This was confirmed by the intracranial field recordings (see Results), which demonstrated fourfold smaller fields in the remote montage than the local montage. To improve skin conductivity at the site of the electrodes, and to reduce the dripping that occurs with saline, we applied a more viscous mixture of water, isopropanol, and aluminum chlorohydrate (Pre-TENS Conductive Skin Preparation, BML Basic Physician's Supply) to the area of the scalp.

\section{Experimental procedures}

In each experiment, the head-fixed monkey started a trial by bringing its gaze within an invisible window of $2 \times 2$ degrees of visual angle surrounding a small red dot that was permanently present at the center of the screen. At the end of a trial, the animals were rewarded with apple juice for maintaining fixation throughout the trial.

In each recording session, we ran two preliminary mapping experiments to guide stimulus location and motion direction of the main experiment. First, we determined the preferred direction (PD) of the neuron using a sparse full-screen pattern of dots that moved along a 
circular path resulting in a uniform translational velocity (Krekelberg, 2008). Second, we determined the spatial receptive field using localized motion pulses in the PD in a matrix of $4 \times 3$ patches covering the screen (Krekelberg and Albright, 2005). In subsequent experiments, the stimuli were centered on the patch that elicited the maximum mean response.

In the main experiment, trials consisted of a $3 \mathrm{~s}$ adapter stimulus followed by a $300 \mathrm{~ms}$ blank period in which only the fixation dot was visible, and a $300 \mathrm{~ms}$ test stimulus. Both the adapter and test stimulus consisted of 700 anti-aliased dots $\left(30 \mathrm{~cd} / \mathrm{m}^{2}\right.$, effective diameter 1.5 pixels) on a $4 \mathrm{~cd} / \mathrm{m}^{2}$ background, moving within a $5^{\circ}$ radius circular aperture.

The main experiment was a $2 \times 2$ factorial design to test the hypothesis that tACS attenuates adaptation induced changes. The first factor was the level of motion adaptation, which we manipulated by choosing the adapter stimulus. Each of the dots in the adapter stimulus either moved in a randomly chosen direction or they all moved in the neuron's PD. The random motion stimulus typically induces less adaptation than the coherent motion stimulus; hence, for ease of reference, we will use the terms "adapted" and "unadapted" for the levels of this factor. The second factor was the presence or absence of tACS; in tACS-ON conditions, it was applied during the $3 \mathrm{~s}$ that the adapter stimulus was on the screen. In the tACS-OFF trials, it was not applied at all.

The dots in the test stimulus moved coherently in 1 of 8 evenly spaced directions spanning the circle. This allowed us to measure a direction tuning curve under each of the experimental conditions. The four conditions of the factorial design were presented in separate blocks with one repeat per test direction. The blocks were randomly interleaved and repeated at least 10 times.

In a separate set of experiments in one animal (Monkey N), we used two intracranial electrodes to measure the induced fields. These electrodes were inserted to the depth of MT in parallel (at a distance of $\sim 1$ $\mathrm{mm}$ in-plane). We then stimulated for $1 \mathrm{~s}$ at $2 \mathrm{~mA}$ peak-to-peak and 10 $\mathrm{Hz}$ through the scalp electrodes and quantified the induced field by extracting the amplitude of the voltage fluctuations at $10 \mathrm{~Hz}$ using the Fourier transform. Using dual electrodes within MT avoided saturation of the amplifiers. The potential difference at the first location in MT was normalized to $0 \mathrm{mV}$. Then, we moved one of the electrodes forward in steps of $100 \mu \mathrm{m}$. Each measurement was repeated 10 times. The electric field, the spatial gradient of the potential, was estimated by fitting a line to the relationship between the potential and the distance between the intracranial electrodes.

\section{Data analysis}

Tuning curves. Our primary interest was to determine how tACS affects direction tuned responses. We used the average response (firing rate) of a neuron during the $300 \mathrm{~ms}$ test interval to estimate tuning curves, separately for each of the four conditions of interest (coherent-adaption/ random-adaptation $\times$ tACS-ON/tACS-OFF). Using a resampling-based Bayesian method (Cronin et al., 2010) we estimated tuning amplitude (TA), tuning width (TW), baseline (untuned) firing rate (UR), and PD of a circular Gaussian tuning curve (see Fig. 2). Statistical tests of changes in these parameters were based on the (absence of) overlap of the confidence intervals estimated by the Bayesian method. The threshold for statistical significance was set to 0.05 .

Correlation analysis. Our behavioral results (Kar and Krekelberg, 2014) show that the influence of tACS depends on the strength of adaptation. To investigate whether a similar relationship holds for the neural effects, we determined the Spearman correlation $(\rho)$ between the change in TA due to tACS $\left(\mathrm{TA}_{\text {adapt }}-\mathrm{TA}_{\text {adapt }}{ }^{\mathrm{tACS}}\right.$ ) and the change in amplitude due to adaptation $\left(\mathrm{TA}_{\text {unadapt }}-\mathrm{TA}_{\text {adapt }}\right)$ (see Fig. 4).

These two measures both depend on $\mathrm{TA}_{\text {adapt }}$ and are thus not mutually independent. This precludes the use of standard significance testing of Spearman's $\rho$. Instead, we used a permutation test. Our observed data (TAs) can be treated as a matrix with $\mathrm{N}$ rows and $\mathrm{M}$ columns, where $\mathrm{N}$ is the number of recorded cells and $\mathrm{M}$ is the number of experimental conditions (column 1: unadapted; column 2: adapted; and column 3: adapted with tACS). Hence, to estimate the significance of $\rho=$ corr (column 1 - column 2, column 2 - column 3 ), we computed the null distribution of correlations by randomly shuffling the data matrix 1000 times (i.e., randomly assigning a new position to each element of the matrix) and estimating $\rho$ for each shuffle. To test for significance, we compared the actual $\rho$ with the 95th percentile of the null distribution. The same analysis method was used for the TW data.

\section{LFP analysis}

Analyses of evoked LFP amplitude. LFPs were bandpass filtered between 1 and $120 \mathrm{~Hz}$ and sampled at $781.25 \mathrm{~Hz}$. The evoked responses were determined by averaging the LFP during test stimulus presentation over all trials corresponding to a given adaptation/stimulation condition. For the average evoked LFP shown in Figure $6 a$, we subtracted the response before stimulus onset (i.e., set the response to zero at time 0 ) and then averaged across all recording sessions. Hence, the curves show the net deflection from baseline following stimulus onset. We observed two pronounced troughs: N1 $(50-70 \mathrm{~ms})$ and N2 $(90-110 \mathrm{~ms})$ in the evoked potential. We performed paired $t$ tests to compare the mean LFP signal in the respective time bands ( $\mathrm{N} 1$ and $\mathrm{N} 2$ ), across conditions.

Spectral analyses of evoked LFP. For the spectral analysis, we focused on the LFP trace recorded $150 \mathrm{~ms}$ after tACS offset (to avoid artifacts from volume conducted stimulation currents). For each recording site, we first calculated the mean evoked LFP for each of the four conditions (coherent-adaption/random-adaptation $\times$ tACS-ON/tACS-OFF) and regressed this out of the raw signal. This allowed us to focus on the induced, rather than evoked oscillations (van Kerkoerle et al., 2014).

Multitaper spectrograms were estimated using used the Chronux software package (Mitra and Bokil, 2008) (http://chronux.org/) with parameters: time bandwidth product $=1.2$, number of tapers $=2$, sampling frequency $=781.25 \mathrm{~Hz}$. We estimated the power spectrum (frequency range $0-120 \mathrm{~Hz}, 40$ nonoverlapping frequency bins) of the LFP for each trial, across all conditions and sites.

Significance of the factors frequency and tACS was assessed using an ANOVA of the normalized power spectrum. In this normalization, the power at each site and frequency was divided by the mean power at that site and frequency, across all experimental conditions. This normalization removed the $1 / \mathrm{f}$ (pink noise) structure of the LFP power spectrum and equated variance across frequency bands.

For Figure 7, we first estimated a tuning curve using the Bayesian method (Cronin et al., 2010) described above, but now based on the LFP power of the $\gamma$ band $(30-120 \mathrm{~Hz})$, which is tuned to motion direction (Liu and Newsome, 2006). The PD of each site was then used to group recordings into 4 bins based on the distance between the direction of the coherent adapter stimulus and the PD of the site.

We also used the Chronux toolbox to determine spike-field coherence (SFC), separately for the frequency bands indicated in the main text. We analyzed the phase of the SFC using a circular two-way ANOVA (Harrison and Kanji, 1988), and the amplitude using a standard two-way ANOVA, both with frequency band and tACS On/Off as the factors.

\section{Results}

tACS electrodes were placed on the shaved scalp over the superior temporal sulcus, one electrode on either side of the implanted recording chamber, close to area MT (Fig. 1a). One or more standard intracranial recording electrodes were lowered into area MT and used to measure the strength of the tACS-induced electric fields as well as the neural activity (see Materials and Methods).

\section{tACS induces intracranial electric fields}

We measured the potential differences induced between two intracranial electrodes in area MT at a range of (intracranial) electrode separations (see Materials and Methods). Figure 1 shows that the potential difference increased linearly with electrode separation, as would be expected in a passive extracellular medium (Logothetis et al., 2007). The electric field magnitude corresponds to the slope of this linear relationship between the potential and the electrode separation. In Figure $1 a$, this results in an estimated field strength of $0.12 \mathrm{~V} / \mathrm{m}\left(R^{2}=0.85\right)$. Figure $1 b$ shows the analogous recording when the tACS electrodes were placed 


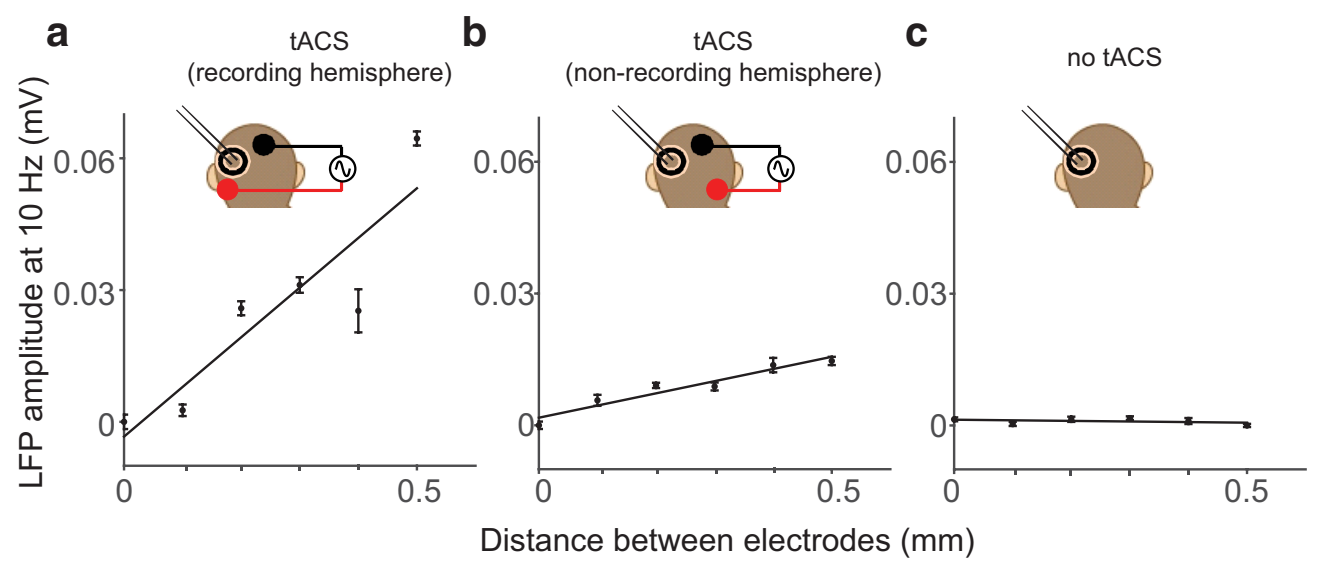

Figure 1. Intracranial electric field measurements. Each panel represents the potential between two intracranial electrodes in area MT. $\boldsymbol{a}$, Potentials during tACS stimulation using the local montage. $\boldsymbol{b}$, Potentials during $\mathrm{tACS}$ using the remote montage. $\boldsymbol{c}$, Potentials in the absence of tACS. This figure represents that local stimulation created a field of $0.12 \mathrm{~V} / \mathrm{m}$ (the slope of the line in a), whereas remote stimulation resulted in only $0.03 \mathrm{~V} / \mathrm{m}$.

over the hemisphere that we did not record from. For this "remote" montage, the induced field was fourfold weaker; $0.03 \mathrm{~V} / \mathrm{m}$ $\left(R^{2}=0.84\right)$. Finally, Figure $1 c$ confirms that the measured fields were indeed caused by tACS; in the absence of tACS, potential differences were an order of magnitude lower and did not vary systematically with electrode separation.

These measurements confirm that $2 \mathrm{~mA}$ peak-to-peak currents applied to the scalp generate fields that are known to be within a range that modulates neural activity in vitro and in vivo (Francis et al., 2003; Ozen et al., 2010). In addition, our finding that tACS induces fourfold smaller fields in the remote montage attests to its (limited) spatial specificity (see Discussion).

\section{tACS affects tuning curves of single neurons}

Having confirmed that tACS generates intracranial electric fields in area MT, we now turn to the question of how these fields change the response properties of individual neurons in area MT. We performed extracellular recordings from 107 motionselective neurons in two male $M$. mulatta. The monkeys were trained to fixate a dot at the center of the screen and maintain fixation while moving random dot stimuli were presented in the neuron's receptive field (Fig. 2a). Based on our previous behavioral findings (Kar and Krekelberg, 2014), we hypothesized that tACS would interfere with the induction of adaptation. We therefore measured direction tuning curves in a strongly adapted state (i.e., after adaptation to $3 \mathrm{~s}$ of coherent motion) and in a weakly adapted state (after adaptation to $3 \mathrm{~s}$ of random motion; we refer to this condition as unadapted; see Materials and Methods). To investigate the influence of tACS on the induction of adaptation, we applied tACS during the adaptation period in half of the trials (chosen randomly). To avoid contamination of the neural signal by potentially lingering stimulation artifacts (Fig. $2 c$ ), we did not analyze the signal recorded during the first $150 \mathrm{~ms}$ after tACS offset (Fig. 2b,c).

\section{Changes in TA}

Figure 3 shows the tuning curves of four example neurons. The TA of the neuron in Figure $3 a$ was much reduced after adaptation to coherent motion (blue curve) compared with after adaptation to random motion (black curve). When coherent-motion adaptation was combined with tACS, the reduction was approximately halved (adapt ${ }^{\mathrm{tACS}}$, red curve). Figure $3 b$ shows an example neuron with a TA that increased after adaptation (increases in firing rate after adaptation are not uncommon in area V1) (Wissig and Kohn, 2012) or MT (Kar and Krekelberg, 2016). In this neuron, the application of tACS during adaptation reduced the increase in firing rate (Fig. $3 b$, red curve). Both examples show that tACS attenuated the effects of adaptation on TA.

At the single-neuron level, adaptation reduced the TA significantly in 23 cells; applying tACS during adaptation increased the TA in 17 of these; and in 7 neurons, the increase due to tACS was individually significant; this confirms that the pattern of changes exemplified by the example neuron in Figure $3 a$ was reliably found in our MT sample. Similarly, 8 cells behaved like the example neuron in Figure $3 b$; they showed significant TA enhancement, and tACS significantly reduced TA in 7 of these (all $p<$ 0.05 ; see Materials and Methods). Additional statistical analysis at the population level (see below; Fig. 4) also confirmed the statistical reliability of the attenuation of adaptation by tACS.

\section{Changes in TW}

The effects of adaptation on the TWs were similarly attenuated by tACS. In Figure $3 c$, tACS reduced the adaptation-induced broadening of the tuning curve, whereas in Figure $3 d$, tACS reduced the adaptation-induced sharpening. When tACS was applied during random-motion adaptation (unadapt ${ }^{\mathrm{tACS}}$, green curve), the changes in TW were smaller and not consistent across cells (Fig. $3 a-d)$. Statistical analysis at the individual neuron level and at the population level (Fig. 4) confirmed the statistical reliability of this finding: 16 cells showed significant TW sharpening and 5 cells showed significant TW broadening. tACS significantly increased the TW in 10 of the 16 sharpened cells and reduced the TW significantly in 3 of the 5 broadened cells (all $p<0.05$; see Materials and Methods).

\section{Untuned responses (URs) and PD}

The UR did not change significantly with adaptation or tACS (paired $t$ test, $p>0.5$ ). Previous work has shown that the PD of a neuron can shift toward the direction of an adapter on the flank of its tuning curve (Kohn and Movshon, 2004). In our sample of MT neurons, we defined neurons as flank-adapted if their PD was within 45 。 to 90 。 from the adapter direction. Within this group $(N=25)$, tuning curves were significantly attracted by the adapter (paired $t$ test, $t_{(25)}=2.25, p=0.01$ ). These attractive shifts, however, were not significantly modulated by tACS (paired $t$ test, $t_{(25)}=0.23, p=0.4$ ). 


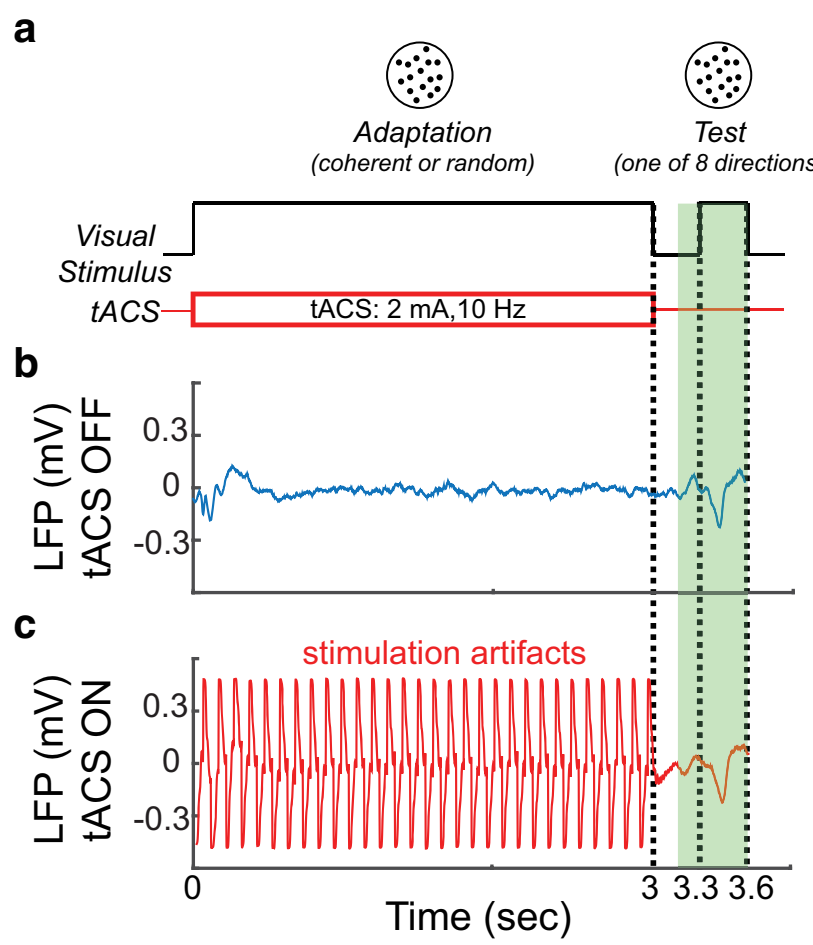

\section{d}

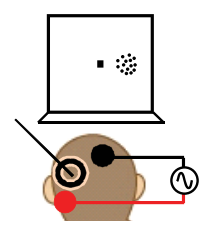

local tACS

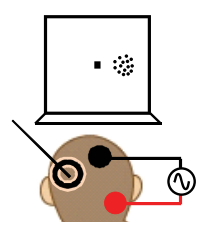

remote tACS

e

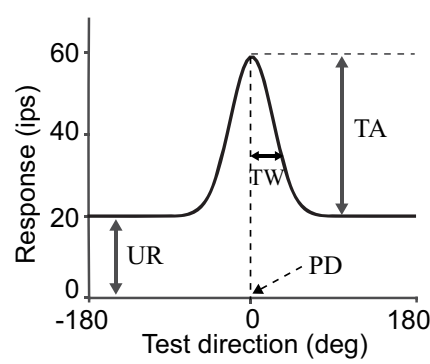

Figure 2. Experimental setup and procedure. $\boldsymbol{a}$, Visual paradigm. On each trial, a dot pattern (random or coherent motion) was presented for $3 \mathrm{~s}$ followed by a blank period of $300 \mathrm{~ms}$, and then a $300 \mathrm{~ms}$ coherent dot pattern (moving in 1 of 8 evenly spaced directions). Monkeys fixated a dot at the center of the monitor. Dot patterns were centered on the receptive field (RF) of the neuron being recorded. $\boldsymbol{b}$, LFPs recorded during an example session without ACS. $\boldsymbol{c}$, LFPs recorded during the same session as $\boldsymbol{b}$ with tACS. LFPs recorded during tACS were dominated by stimulation artifacts. Hence, we only analyzed data obtained at least $150 \mathrm{~ms}$ after tACS offset (green shading). $\boldsymbol{d}$, Schematic of the local and remote tACS conditions. $\boldsymbol{e}$, Tuning curve definition. Each tuning curve was described with four parameters: UR, TA, TW, and PD. ips, Impulses per second.

The effects of tACS are proportional to the level of adaptation To determine whether the attenuation of adaptation by tACS was consistent across the population, we compared the four TAs and widths obtained in each of the neurons in our sample. Figure $4 a$ shows the relation between the adaptation-induced change in amplitude and the effect of tACS. There was a significant negative correlation (Spearman correlation, $\rho_{(107)}=-0.61, p<0.001$, permutation test; see Materials and Methods), which shows that that tACS significantly attenuated the effect of adaptation regardless of the sign of the adaptation effect. Figure $4 b$ shows the analogous analysis for changes in the TWs. In neurons whose tuning was broadened by adaptation, tACS led to a narrowing of the tuning, and in neurons whose tuning curves became narrower after adaptation, tACS led to a broadening. This correlation was also significantly negative (Spearman correlation, $\rho_{(107)}=-0.7$, $p<0.001)$ and provides additional support for the hypothesis that tACS attenuates adaptation.

An alternative hypothesis for the tACS-induced changes in tuning curves could be that tACS produces unspecific changes that are unrelated to adaptation, for instance, general changes in arousal or attentional signals that drive the responses up or down. If this were the case, one would expect the changes to be similar in the adapted and the unadapted conditions. Our data do not support this hypothesis. Figure $4 c, d$ shows that, across the sample of neurons in which we recorded both unadapted and adapted conditions $(N=93)$, the tACS-induced changes in the unadapted conditions were not correlated with the changes in the adapted conditions $\left(\mathrm{TA}: \rho_{(93)}=-0.01, p=0.51\right.$; TW: $\rho_{(93)}=-0.06$, $p=0.84)$.

To provide further evidence that the effects of tACS are spatially specific and cannot be attributed to general arousal effects, we performed an experiment in which tACS could be applied to either hemisphere (Fig. $2 d$ ). In one random subset of trials, we passed the alternating current through electrodes around the MT recording chamber (local montage), and in the other trials we passed the same current through electrodes positioned on the mirrored locations on the opposite hemisphere (remote montage). As our field recordings in Figure $1 a, b$ show, the latter montage produced approximately fourfold weaker electric fields. In the local stimulation conditions, this experiment replicated our finding (Fig. 5a) that tACS attenuated adaptation, and that this effect increased with the strength of adaptation (black line; Spearman correlation, $\left.\rho_{(21)}=-0.65, p<0.001\right)$. Importantly, the remote stimulation condition did not result in a significantly negative correlation (red line; $\rho_{(21)}=-0.06, p=0.6$ ). A direct comparison of the local and remote conditions confirmed that they were significantly different $(p<0.05$, permutation test) and the difference between the effect of local and remote tACS was positively correlated (Fig. $5 b$ ) with the strength of adaptation on a cell-by-cell basis $\left(\rho_{(21)}=0.5, p=0.01\right)$. Together, these data show that the attenuation of adaptation of single neurons is a spatially specific effect of tACS that cannot be attributed to global changes in arousal or attention.

\section{tACS modulates evoked LFPs}

We used the LFPs recorded at 75 sites in area MT of Monkey N (59) and Monkey M (17) to gain insight into tACS-induced changes in aggregate population activity. Figure $6 a$ shows the evoked, baseline-corrected, LFPs averaged over recording sites (see Materials and Methods). For completeness, there was no significant difference in the baseline responses (100 ms before the 
test stimulus onset) between the four conditions (data not shown).

The evoked responses to the adapted and unadapted test stimulus (solid black and blue curve) were similar. We attribute this to the fact that many neurons, with a potentially large range of PDs, contributed to these evoked responses. Hence, a random motion stimulus that weakly adapted many neurons could result in a similar change as a coherent motion stimulus that strongly adapted a subset of the neurons. In addition, adaptation effects that are not specific to the direction of motion (e.g., contrast adaptation) would sum in the large population that contributes to the LFP, and dominate the direction-specific effects we see more clearly in the single-neuron responses. In other words, the terms "adapted" and "unadapted," although commonly used for the analysis of single units, are somewhat of a misnomer for the LFP analysis; both responses to the test stimulus are indeed "adapted."

We confirmed this by analyzing the response to the adapter stimulus (Fig. $6 a$, dashed black curve). Because the adapter was always presented at the start of a trial, it appeared after a period of very low visual stimulation; the intertrial interval during which the animal would also often blink or move its eyes. In other words, this response to the first stimulus in a trial provided an estimate of a truly unadapted evoked LFP response. Comparing this unadapted evoked LFP response (unadapt ${ }^{\text {PRE }}$, Fig. $6 a$, dashed black curve) with the test-evoked responses for directions within $45^{\circ}$ of the adapter direction (Fig. $6 a$, solid black and blue curves) shows that adaptation led to a reduced evoked response. Consistent with findings in visually evoked potentials in humans (Hoffmann et al., 1999), adaptation led to a statistically significant reduction in the first and second negativity, N1 (50-70 ms, average suppression $\left.0.025 \mathrm{mV} ; t_{(75)}=1.67, p<0.001\right)$ and $\mathrm{N} 2(90-110 \mathrm{~ms}$, average suppression $0.017 \mathrm{mV} ; t_{(76)}=1.54, p<0.001$ ), respectively.

The test-evoked responses in the tACS trials (red and green curves) show two distinct effects. First, the test-evoked N2 component in the tACS trials was as strong as the adapter-evoked N2 (dashed curves). In other words, tACS significantly attenuated the effect of adaptation on the N2 component (average attenuation $\left.0.03 \mathrm{mV} ; t_{(75)}=-2.33, p=0.01\right)$. Second, tACS significantly increased (average increase $0.04 \mathrm{mV} ; t_{(75)}=3.14, p=0.001$ ) the later part of the evoked potential ( $>100 \mathrm{~ms}$ after stimulus onset). In this phase, adaptation had little effect (i.e., the blue, black, and dashed black curves overlap); hence, this particular effect demonstrates that at least some tACS-induced neural changes do not require strong adaptation.

\section{tACS increases broadband spectral power}

Next, we investigated the influence of $10 \mathrm{~Hz}$ tACS on the aggregate population activity reflected in the LFP. To avoid contamination by stimulation artifacts, we only considered LFPs recorded at least $150 \mathrm{~ms}$ after the offset of stimulation. Furthermore, to focus on induced oscillations, we regressed out the av- erage evoked response (i.e., the data leading to Fig. $6 a$ ) from each trial before calculating the power per frequency band (see Materials and Methods). The power in the induced LFP is shown in Figure $6 b$. The expected 1/f power drop-off is clearly visible in all conditions. Another notable feature is that there appears to be no peak at $10 \mathrm{~Hz}$ either before or after tACS (see Discussion).

The LFP power of the response to the adapter stimulus (black dashed curve) was higher than the power of the response to the test stimuli (blue and black curves) across the spectrum. As in the evoked potential, this difference was similar regardless of whether the adapter was a coherent motion stimulus (blue curve) or a random motion stimulus (black curve). In other words, adaptation reduced broadband spectral power. Figure $6 b$ (red and green curves) shows that the spectral power in response to the test stimulus was larger when tACS had been applied during the adaptation phase. This broadband increase in power did not depend on the removal of the evoked response (see Materials and Methods), or even the presence of a visual stimulus, as qualitatively similar increases were observed in a spectral analysis of the raw LFP as well as the spontaneous LFP recorded in the $150 \mathrm{~ms}$ before the onset of the test stimulus (data not shown).

Statistically, we first normalized the power to the mean power across conditions and then used a three-way repeated-measures (sites) ANOVA with factors of adaptation (coherent/random), stimulation (tACS/no-tACS), and LFP frequency (see Materials and Methods). There were significant main effects of adaptation $\left(F_{(2,1)}>28, p<0.001\right)$ as well as stimulation $\left(F_{(2,1)}>45, p<\right.$ 

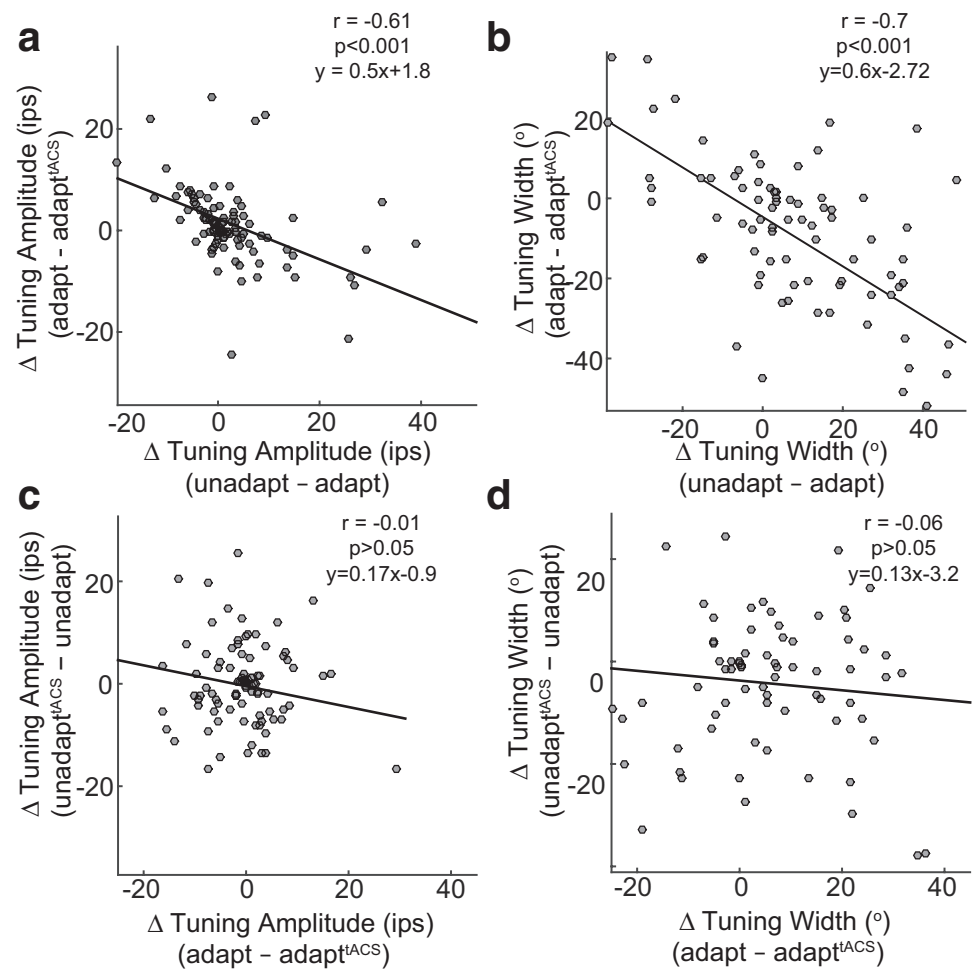

Figure 4. Population analysis of tACS-induced changes in tuning properties. $\boldsymbol{a}$, Comparison of the change in TA induced by adaptation during $\mathrm{tACS}$ with the change in $\mathrm{TA}$ induced by adaptation alone. Each dot represents a single neuron. Lines indicate the result of an orthogonal linear regression fit (parameters written as text on panel). $\boldsymbol{b}$, Same as $\boldsymbol{a}$, but comparing changes in TW. $\boldsymbol{c}$ Comparison of the tACS-induced change in TA in the unadapted conditions with tACS-induced change in TA in the adapted conditions. $\boldsymbol{d}$, Same as $\boldsymbol{c}$, but comparing changes in TW. This figure represents that the tuning curve changes induced by adaptation alone (and only those changes) are partially undone when adaptation is combined with tACS. In other words, tACS consistently attenuated adaptation.

$0.001)$ both during and before test stimulus presentation, but there were no main or interaction effects of LFP frequency $\left(F_{(2,39)}\right.$ $<1, p>0.05)$ on the normalized power. During test stimulus presentation, there was a significant interaction between adaptation and stimulation $\left(F_{(2,1)}=131, p<0.001\right)$, but this interaction was not significant before the presentation of the test stimulus $\left(F_{(2,1)}=0.56 ; p=0.45\right)$.

To further investigate the dependence of this power increase during test stimulus presentation on adaptation, we separated recording sites on the basis of their PD (the circular mean of the gamma tuning curve, i.e., the power measured in the $\gamma$ band, $30-120 \mathrm{~Hz}$, as a function of motion direction; see Materials and Methods). Figure $7 a$ shows the broadband $(0-120 \mathrm{~Hz})$ LFP power was larger in the unadapted compared with the adapted conditions ( $\Delta$ Power $>0$ ). Consistent with previous reports that spike rate adaptation is stronger when sites are adapted near their preferred stimulus (Kohn and Movshon, 2004; Krekelberg et al., 2006; Kar and Krekelberg, 2016), sites adapted near their PD adapted most. Figure $7 b$ shows the influence of tACS on the LFP power. tACS increased LFP power most in those sites in which adaptation reduced the power most.

\section{Short-term tACS did not evoke long-lasting frequency-specific entrainment}

Previous work in the slice (Francis et al., 2003), in rats (Ozen et al., 2010), in ferrets (Fröhlich and McCormick, 2010), and in humans (Helfrich et al., 2014) has shown that neural oscillations can be entrained by applied fields. Several analyses of our data suggest that there was no $10 \mathrm{~Hz}$ specific entrainment during test stimulus presentation in our data. Although our stimulation protocol differed substantially from previous studies, and these negative results should be interpreted with caution (see Discussion); we list them here.

First, visual inspection of the power spectra after stimulation in Figure $6 b$ shows that they do not exhibit a peak at 10 $\mathrm{Hz}$. Second, we confirmed this statistically by the lack of a significant interaction between LFP frequency and the effect of tACS (see ANOVA above). Third, to increase the power of the analysis and zoom in on the relevant frequency bands, we divided the LFP frequencies into five nonoverlapping bands: $\alpha(8-15 \mathrm{~Hz}), \beta$ (15-30 Hz), low $\gamma(30-50 \mathrm{~Hz})$, medium $\gamma$ $(50-80 \mathrm{~Hz})$, and high $\gamma(80-120 \mathrm{~Hz})$. We then calculated the ratio of the power in the tACS conditions with the corresponding no-tACS conditions per frequency band. This approximately equalized the variance across conditions and enabled us to perform a one-way ANOVA with frequency band as a factor. The main effect of frequency was not significant either before or during test stimulus presentation $\left(F_{(4)}<0.001, p>0.05\right)$. This further confirms that the increase in spectral power was indeed broadband. Finally, we considered whether entrainment of neural activity occurred by increased phaselocking of spikes relative to the phase of the LFP. We calculated this SFC separately for the five frequency bands, in the tACS and no-tACS conditions, and before or during test stimulus onset. Two-way ANOVAs on the phase and amplitude of the SFC showed no significant effect of tACS $\left(F_{(1)}<1\right.$, $p>0.05)$, nor a significant interaction between tACS and frequency $\left(F_{(2,4)}<2, p>0.05\right)$. Together, these results show that the short $(3 \mathrm{~s})$ bursts of $10 \mathrm{~Hz}$ tACS did not generate entrainment in a sustained and stimulation frequency-specific manner (see Discussion).

\section{Discussion}

Our data reveal, for the first time, a number of ways in which tACS affects neural activity in the brains of awake, behaving primates. Several findings support the hypothesis that tACS interferes with the mechanisms of adaptation. First, we found that tACS attenuated the effects of visual motion adaptation on the amplitude and width of the tuning curves of single neurons. The impact of tACS was strongest on neurons that adapted most. Moreover, the effect was specific to the montage in which the stimulation electrodes were on the same hemisphere as the recorded neurons. This both attests to the spatial specificity of the tACS effect and excludes potential confounds of changes in arousal. Second, we found that tACS attenuated the adaptationinduced reduction of the N2 component of the evoked LFP. Finally, we found broadband increases in spectral power that persisted for at least $300 \mathrm{~ms}$ after tACS offset. This effect was also most pronounced at sites where adaptation was strongest.

We first discuss a number of potential confounds, compare our results with previous studies, assess how these findings im- 
pact the use of tACS, and finally speculate on the cellular mechanisms that may underlie these effects.

\section{Potential confounds}

It is possible that stimulation induced a behavioral change (unrelated to the neural activity in area MT) and that this behavioral change resulted in the neural changes in MT. In our experiments, however, behavior was tightly controlled; trials in which the animal blinked, or made large eye movements, were excluded from the analysis. For completeness, we also analyzed whether any of these events were more frequent during tACS, but we found no evidence to support this claim.

Phosphenes are another potential confound in many tACS studies. These shimmering sensations are easily evoked by 2 mA peak-to-peak tACS at $10 \mathrm{~Hz}$ (Rohracher, 1935), and most likely have a retinal origin (Schwiedrzik, 2009; Schutter and Hortensius, 2010; Kar and Krekelberg, 2012; Laakso and Hirata, 2013). Phosphenes are typically perceived in the periphery of the visual field, which limits their interaction with our parafoveal motion stimuli. Nonetheless, they could draw attention away from the motion stimuli and thereby reduce neural responses (Martínez-Trujillo and Treue, 2002) and adaptation (Dobkins et al., 2007). However, the clear difference between remote and local stimulation (Fig. 1) argues against this interpretation and in favor of a specific influence of tACS on neuronal adaptation in the stimulated hemisphere.

\section{Electric field estimates}

Our measurement of the intracranial electric field generated by 2 $\mathrm{mA}$ peak-to-peak tACS shows that it is of an order of magnitude that is sufficient to modulate neural activity (Francis et al., 2003; Ozen et al., 2010). It is reassuring to note that the fields we measured in the macaque brain are within the range predicted by current flow models based on human anatomy (Miranda et al., 2006). Of course, the macaque head is smaller than the human head, and the surgical preparation for intracranial recordings inevitably changes current flow; hence, this only provides a coarse (order of magnitude) validation of these models. Given the potential importance of delivering specific doses of electric fields to targeted areas of the brain, more quantitative validation of current flow models that take idiosyncrasies in scalp, skull, and cortical folding into account, remains necessary (Dmochowski et al., 2011).

\section{Entrainment}

Part of the appeal of tACS is that temporal patterns of stimulation can be modulated at will, and can potentially target different oscillations. Previous animal studies have provided direct experimental evidence that tACS can entrain neural activity (Ozen et al., 2010; Ali et al., 2013). This is a potentially useful feature of tACS as task-relevant stimulation frequencies could modulate specific behavior (Joundi et al., 2012; Polanía et al., 2012; Strüber et al., 2014; Voss et al., 2014).

In our data, we found no evidence of stimulation frequencyspecific entrainment. Many factors could have contributed to this, and our negative findings should not be misconstrued as a conflict with previous studies. First and foremost, because amplifier saturation prevented us from reliably recording neural activity during stimulation, we only analyzed entrainment after tACS offset. Previous animal studies also reported no entrainment after stimulation offset, which is consistent with our findings. Second, compared with previous studies in humans, our stimulation epochs were short: 3 s compared with $20 \mathrm{~min}$ in Helfrich et al. (2014). This suggests that longer stimulation periods are needed to generate entrainment that outlasts the stimulation period, and there is some direct evidence to support this (Kar, 2015; Strüber et al., 2015). Finally, unlike in previous studies in humans (e.g., Helfrich et al., 2014), the power spectrum before stimulation in our study did not contain a conspicuous peak at $10 \mathrm{~Hz}$ (Fig. 6). This could also limit entrainment as it may be easier for tACS to boost an already present rhythm than establish one de novo (Ali et al., 2013; Vossen et al., 2015).

We did observe some clear changes in LFP spectral power, even $>300 \mathrm{~ms}$ after the offset of tACS. This increase, however, was not specific to the $10 \mathrm{~Hz}$ stimulation frequency but observed in the entire frequency range. Hence, it should not be interpreted as a form of entrainment. As shown in Figure 6, adaptation resulted in a broadband decrease in LFP power, and tACS attenuated that decrease. Based on that finding, our interpretation is that this broadband increase in spectral power seen when tACS is applied during adaptation is an indirect consequence of the attenuation of adaptation.

\section{Attenuating adaptation}

We identified a novel mechanism by which tACS affects neural activity: the attenuation of adaptation. This finding speaks to the mechanisms of tACS but can potentially also guide how tACS will be used in future applications.

The attenuation of adaptation hypothesis provides insight into the question how tACS can have specific behavioral effects even though its spatial specificity is limited, especially when using standard large-pad electrodes (Laakso and Hirata, 2013). If tACS primarily affects adaptation, then it will affect only those neurons that are undergoing adaptation at the time of tACS stimulation. This novel insight has practical implications for the clinical usage 

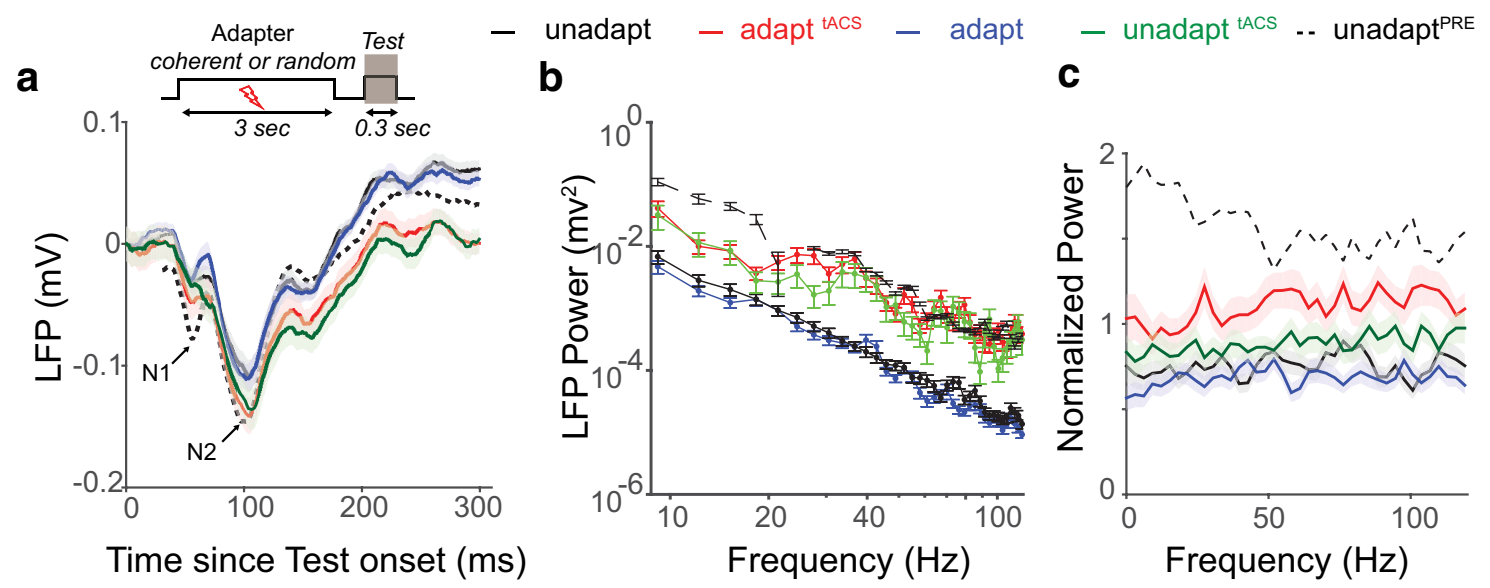

Figure 6. The influence of tACS on LFPs. $\boldsymbol{a}$, The LFP response evoked by the adapter (dashed, unadapt ${ }^{\mathrm{PRE}}$ ) and the test stimuli: solid, unadapt (black), adapt (blue), unadapt ${ }^{\text {tACS }}$ (green), and adapt $^{\text {tACS }}$ (green). Data were averaged $>76$ sites. Adaptation reduced the N1 and N2 components (compare dashed black curve with solid black and blue curves). tACS attenuated the reduction of the N2 component and also increased the magnitude of the evoked LFP after $100 \mathrm{~ms}$ (compare red and green curves with the dashed black curve). $\boldsymbol{b}$, Power spectrum of the LFP (see Materials and Methods). LFP power was significantly reduced in the test interval (compare dashed black curve with solid blue and black curves), but tACS attenuated this reduction (red and green curves). c, Normalized power spectrum of the LFP (see Materials and Methods). LFP power was significantly reduced in the test interval (compare dashed black curve with solid blue and black curves), but tACS attenuated this reduction (red and green curves). There was no evidence for a frequency-specific (i.e., $10 \mathrm{~Hz}$ ) entrainment of the LFP during the test stimuli. Shaded areas and error bars represent SEM.

a

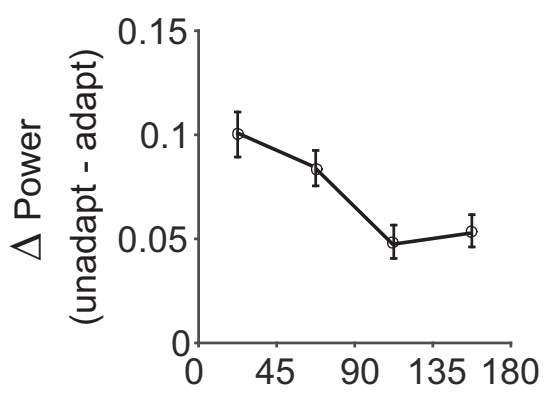

Adapted - Pref. direction

(deg)

b

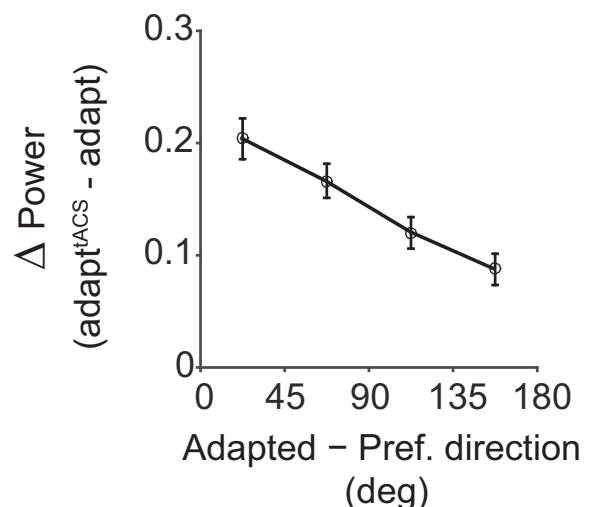

Figure 7. Changes in broadband LFP power after adaptation and tACS. $\boldsymbol{a}$, Change in broadband LFP power after adaptation, as a function of the difference between the sites' PD (see Materials and Methods) and the adapters' direction of motion. Sites that were adapted near their PD of motion showed a greater decrease in broadband spectral power. $\boldsymbol{b}$, Change in LFP power due to $\mathrm{tACS}$ applied during the adaptation phase. $\mathrm{ACS}$ increased power most in sites that adapted most. Error bars indicate SE.

of tACS because it implies that the subset of neurons affected by tACS can be changed by adjusting the task that the subject performs while undergoing tACS. In other words, to target a brain area, one can choose to adapt it with a relevant stimulus or task, which will make it more susceptible to tACS.
The relatively slow time scale of adaptation provides a pathway for tACS effects to become longer lasting. When the transcranial current is turned off, the imposed intracranial fields vanish instantly. Hence, if tACS only affected membrane polarization, then its effects should dissipate on a time scale on the order of at most a few tens of milliseconds (the membrane time constant). If, however, tACS also affects the cellular mechanisms underlying adaptation, then its effects will be felt on a time scale that extends beyond tACS offset. Here we used an adaptation protocol that typically induces changes on a time scale of seconds, but in future work, we plan to investigate whether a further extension in time scale can be achieved by tACS' interaction with longer lasting adaptation.

We do not claim that the only consequence of $10 \mathrm{~Hz}$ tACS is an attenuation of adaptation. As discussed above, entrainment may well have occurred during stimulation. In addition, the influence on the late evoked response ( $>100 \mathrm{~ms}$; Fig. 6) is opposite to the influence of adaptation, suggesting that a separate mechanism may be responsible for this effect.

Conversely, we also do not claim that only $10 \mathrm{~Hz}$ stimulation attenuates adaptation. We chose this frequency mainly to match our human behavioral experiments, but other frequencies and even DC stimulation (Antal et al., 2004) could also attenuate adaptation. However, it is known that sensitivity of the neuronal transmembrane potential to the applied external field drops exponentially with frequency (Deans et al., 2007). This limits the likely efficacy of high-frequency stimulation. More specifically, if our hypothesis about the underlying cellular mechanisms is correct (see below), stimulation in the $10-20 \mathrm{~Hz}$ range should be most effective (Fernandez et al., 2011). Hence, even though other stimulation frequencies could have similar effects, the practical constraints of single electrode, single-unit recordings in awake, behaving animals led us to focus exclusively on $10 \mathrm{~Hz}$ stimulation. In future work, we plan to explore a wider range of frequencies and stimulation patterns using the longer recording times made possible by permanently implanted electrode arrays. 


\section{Cellular mechanisms}

The small intracranial fields induced by transcranial stimulation has led to some skepticism about its efficacy (Horvath et al., 2015). Our results suggest a possible resolution of this issue because even small $(<2 \mathrm{mV})$ membrane voltage fluctuations, especially in the $10-20 \mathrm{~Hz}$ range, have been shown to reduce spike frequency adaptation in rat hippocampal CA1 neurons by preventing the opening of sodium-dependent potassium channels (Fernandez et al., 2011). Based on these in vitro data, we speculate that, in our recordings of MT neurons, the tACS-induced membrane-potential fluctuations may have prevented the activation of sodium- or calcium-dependent potassium channels. This would attenuate spike rate adaptation (Sanchez-Vives et al., 2000), resulting in much larger changes in firing rate than may be expected from a few millivolts of membrane polarization. This is a novel mode of action for tACS; it relies neither on bringing the membrane potential closer to threshold nor on the entrainment of networks of neurons. But by tapping into cellular level mechanisms that regulate adaptation, it can nevertheless exert a potent and relatively long-lasting influence on the network. This novel hypothesis could be tested with in vitro cortical slice recordings.

\section{References}

Ali MM, Sellers KK, Fröhlich F (2013) Transcranial alternating current stimulation modulates large-scale cortical network activity by network resonance. J Neurosci 33:11262-11275. CrossRef Medline

Antal A, Varga ET, Nitsche MA, Chadaide Z, Paulus W, Kovács G, Vidnyánszky Z (2004) Direct current stimulation over $\mathrm{MT}^{+} / \mathrm{V} 5$ modulates motion aftereffect in humans. Neuroreport 15:2491-2494. CrossRef Medline

Born RT, Bradley DC (2005) Structure and function of visual area MT. Annu Rev Neurosci 28:157-189. CrossRef Medline

Cronin B, Stevenson IH, Sur M, Körding KP (2010) Hierarchical Bayesian modeling and Markov chain Monte Carlo sampling for tuning-curve analysis. J Neurophysiol 103:591-602. CrossRef Medline

Datta A, Bansal V, Diaz J, Patel J, Reato D, Bikson M (2009) Gyri-precise head model of transcranial direct current stimulation: improved spatial focality using a ring electrode versus conventional rectangular pad. Brain Stimul 2:201-207. CrossRef Medline

Deans JK, Powell AD, Jefferys JG (2007) Sensitivity of coherent oscillations in rat hippocampus to AC electric fields. J Physiol 583:555-565. CrossRef Medline

Dmochowski JP, Datta A, Bikson M, Su Y, Parra LC (2011) Optimized multi-electrode stimulation increases focality and intensity at target. J Neural Eng 8:046011. CrossRef Medline

Dobkins KR, Rezec AA, Krekelberg B (2007) Effects of spatial attention and salience cues on chromatic and achromatic motion processing. Vision Res 47:1893-1906. CrossRef Medline

Fernandez FR, Broicher T, Truong A, White JA (2011) Membrane voltage fluctuations reduce spike frequency adaptation and preserve output gain in CA1 pyramidal neurons in a high-conductance state. J Neurosci 31: 3880-3893. CrossRef Medline

Francis JT, Gluckman BJ, Schiff SJ (2003) Sensitivity of neurons to weak electric fields. J Neurosci 23:7255-7261. Medline

Fröhlich F, McCormick DA (2010) Endogenous electric fields may guide neocortical network activity. Neuron 67:129-143. CrossRef Medline

Harris KD, Henze DA, Csicsvari J, Hirase H, Buzsáki G (2000) Accuracy of tetrode spike separation as determined by simultaneous intracellular and extracellular measurements. J Neurophysiol 84:401-414. Medline

Harrison D, Kanji GK (1988) The development of analysis of variance for circular data. J Appl Stat 15:197-233. CrossRef

Helfrich RF, Schneider TR, Rach S, Trautmann-Lengsfeld SA, Engel AK, Herrmann CS (2014) Entrainment of brain oscillations by transcranial alternating current stimulation. Curr Biol 24:333-339. CrossRef Medline

Herrmann CS, Rach S, Neuling T, Strüber D (2013) Transcranial alternating current stimulation: a review of the underlying mechanisms and modulation of cognitive processes. Front Hum Neurosci 7:279. CrossRef Medline

Hoffmann M, Dorn TJ, Bach M (1999) Time course of motion adaptation: motion-onset visual evoked potentials and subjective estimates. Vision Res 39:437-444. CrossRef Medline

Horvath JC, Forte JD, Carter O (2015) Quantitative review finds no evidence of cognitive effects in healthy populations from single-session transcranial direct current stimulation (tDCS). Brain Stimul 8:535-550. CrossRef Medline

Joundi RA, Jenkinson N, Brittain JS, Aziz TZ, Brown P (2012) Driving oscillatory activity in the human cortex enhances motor performance. Curr Biol 22:403-407. CrossRef Medline

Kadir SN, Goodman DF, Harris KD (2014) High-dimensional cluster analysis with the masked EM algorithm. Neural Comput 26:2379-2394. CrossRef Medline

Kar K (2015) Commentary: on the possible role of stimulation duration for after-effects of transcranial alternating current stimulation. Front Syst Neurosci 9:148. CrossRef Medline

Kar K, Krekelberg B (2012) Transcranial electrical stimulation over visual cortex evokes phosphenes with a retinal origin. J Neurophysiol 108:21732178. CrossRef Medline

Kar K, Krekelberg B (2014) Transcranial alternating current stimulation attenuates visual motion adaptation. J Neurosci 34:7334-7340. CrossRef Medline

Kar K, Krekelberg B (2016) Testing the assumptions underlying fMRI adaptation using intracortical recordings in area MT. Cortex 80:21-34. CrossRef Medline

Kohn A, Movshon JA (2004) Adaptation changes the direction tuning of macaque MT neurons. Nat Neurosci 7:764-772. CrossRef Medline

Krekelberg B (2008) Perception of direction is not compensated for neural latency. Behav Brain Sci 31:208-209.

Krekelberg B, Albright TD (2005) Motion mechanisms in macaque MT. J Neurophysiol 93:2908-2921. CrossRef Medline

Krekelberg B, van Wezel RJ, Albright TD (2006) Adaptation in macaque MT reduces perceived speed and improves speed discrimination. J Neurophysiol 95:255-270. CrossRef Medline

Laakso I, Hirata A (2013) Computational analysis shows why transcranial alternating current stimulation induces retinal phosphenes. J Neural Eng 10:046009. CrossRef Medline

Liu J, Newsome WT (2006) Local field potential in cortical area MT: stimulus tuning and behavioral correlations. J Neurosci 26:7779-7790. CrossRef Medline

Logothetis NK, Kayser C, Oeltermann A (2007) In vivo measurement of cortical impedance spectrum in monkeys: implications for signal propagation. Neuron 55:809-823. CrossRef Medline

Martínez-Trujillo J, Treue S (2002) Attentional modulation strength in cortical area MT depends on stimulus contrast. Neuron 35:365-370. CrossRef Medline

Miranda PC, Lomarev M, Hallett M (2006) Modeling the current distribution during transcranial direct current stimulation. Clin Neurophysiol 117:1623-1629. CrossRef Medline

Mitra P, Bokil H (2008) Observed brain dynamics. Oxford: Oxford UP.

Orban GA, Fize D, Peuskens H, Denys K, Nelissen K, Sunaert S, Todd J, Vanduffel W (2003) Similarities and differences in motion processing between the human and macaque brain: evidence from fMRI. Neuropsychologia 41:1757-1768. CrossRef Medline

Ozen S, Sirota A, Belluscio MA, Anastassiou CA, Stark E, Koch C, Buzsáki G (2010) Transcranial electric stimulation entrains cortical neuronal populations in rats. J Neurosci 30:11476-11485. CrossRef Medline

Parker AJ, Newsome WT (1998) Sense and the single neuron: probing the physiology of perception. Annu Rev Neurosci 21:227-277. CrossRef Medline

Polanía R, Nitsche MA, Korman C, Batsikadze G, Paulus W (2012) The importance of timing in segregated theta phase-coupling for cognitive performance. Curr Biol 22:1314-1318. CrossRef Medline

Priebe NJ, Churchland MM, Lisberger SG (2002) Constraints on the source of short-term motion adaptation in macaque area MT: I. The role of input and intrinsic mechanisms. J Neurophysiol 88:354-369. CrossRef Medline

Reato D, Rahman A, Bikson M, Parra LC (2013) Effects of weak transcranial alternating current stimulation on brain activity: a review of known mechanisms from animal studies. Front Hum Neurosci 7:687. CrossRef Medline

Rohracher H (1935) Uber subjektive Lichterscheinungen bei Reizung mit Wechselstromen. Z Sinnesphysiologie 66:164-181.

Sanchez-Vives MV, Nowak LG, McCormick DA (2000) Membrane mecha- 
nisms underlying contrast adaptation in cat area 17 in vivo. J Neurosci 20:4267-4285. Medline

Schlack A, Krekelberg B, Albright TD (2007) Recent history of stimulus speeds affects the speed tuning of neurons in area MT. J Neurosci 27: 11009-11018. CrossRef Medline

Schutter DJ, Hortensius R (2010) Retinal origin of phosphenes to transcranial alternating current stimulation. Clin Neurophysiol 121:1080-1084. CrossRef Medline

Schwiedrzik CM (2009) Retina or visual cortex? The site of phosphene induction by transcranial alternating current stimulation. Front Integr Neurosci 3:6. CrossRef Medline

Sela T, Kilim A, Lavidor M (2012) Transcranial alternating current stimulation increases risk-taking behavior in the balloon analog risk task. Front Neurosci 6:22. CrossRef Medline

Strüber D, Rach S, Trautmann-Lengsfeld SA, Engel AK, Herrmann CS (2014) Antiphasic $40 \mathrm{~Hz}$ oscillatory current stimulation affects bistable motion perception. Brain Topogr 27:158-171. CrossRef Medline

Strüber D, Rach S, Neuling T, Herrmann CS (2015) On the possible role of stimulation duration for after-effects of transcranial alternating current stimulation. Front Cell Neurosci 9:311. CrossRef Medline

van Kerkoerle T, Self MW, Dagnino B, Gariel-Mathis MA, Poort J, van der
Togt C, Roelfsema PR (2014) Alpha and gamma oscillations characterize feedback and feedforward processing in monkey visual cortex. Proc Natl Acad Sci U S A 111:14332-14341. CrossRef Medline

Voss U, Holzmann R, Hobson A, Paulus W, Koppehele-Gossel J, Klimke A, Nitsche MA (2014) Induction of self awareness in dreams through frontal low current stimulation of gamma activity. Nat Neurosci 17:810-812. CrossRef Medline

Vossen A, Gross J, Thut G (2015) Alpha power increase after transcranial alternating current stimulation at alpha frequency (alpha-tACS) reflects plastic changes rather than entrainment. Brain Stimul 8:499-508. CrossRef Medline

Wagner T, Fregni F, Fecteau S, Grodzinsky A, Zahn M, Pascual-Leone A (2007) Transcranial direct current stimulation: a computer-based human model study. Neuroimage 35:1113-1124. CrossRef Medline

Wissig SC, Kohn A (2012) The influence of surround suppression on adaptation effects in primary visual cortex. J Neurophysiol 107:3370-3384. CrossRef Medline

Wright JM, Krekelberg B (2014) Transcranial direct current stimulation over posterior parietal cortex modulates visuospatial localization. J Vis 14:pii5. CrossRef Medline 